\title{
Research on the Current Situation and Future Trend of Web Celebrity E-commerce Live Streaming Industry
}

\author{
Jinqi Zhang ${ }^{1, *}$ \\ ${ }^{1}$ New Chanel Jinqiu institute of Hangzhou, Hangzhou, Zhejiang 310003, China \\ ${ }^{*}$ Corresponding author. Email: zjq15068157566@163.com
}

\begin{abstract}
This paper first explains the specific definition of live e-commerce, and further combs the development process of the industry. In terms of current situation, the author analyzes China's online Red economy live e-commerce industry from both sides of demand and supply. On the demand side, this paper analyzes the live broadcast industry from the policy, economic and other driving forces, and calculates the market scale according to the available data. On the supply side, this paper draws the industrial chain structure chart, summarizes and analyzes all kinds of participants in the industry, and makes data analysis on business model and value. In addition, the author takes Taobao as an example to analyze the typical cases of live e-commerce. On this basis, this paper discusses the future development trend of ecommerce live broadcasting industry, in order to provide suggestions and suggestions for relevant departments and practitioners of live broadcasting industry.
\end{abstract}

Keywords: Web Celebrity Economy, Live Streaming, E-commerce, Business Model, MCN

\section{INTRODUCTION}

According to the data jointly released by Taobao and Taobao live broadcast, the turnover of Tmall on the day of double 11 in 2020 reached 498.2 billion CNY, of which, the growth rate of GMV of Taobao live broadcast exceeded $100 \%$, and the transaction volume of more than 30 Taobao live broadcast rooms exceeded RMB 100 million. Under the background of covid-19 impact on the consumer industry, the value has attracted public attention again, and the online Red live broadcasting industry is becoming more and more attractive. Live e-commerce refers to the form of ecommerce which aims to achieve marketing purpose through live broadcast, and is the product of the integration of live broadcast and e-commerce in the background of digital era. In March 2016, Mushroom Street officially went online to live broadcast entrance, and China's live video business started from this. In the five years of rapid development, many platforms have successively entered the Bureau live e-commerce industry. The specific differences between traditional ecommerce and live e-commerce are shown in Table 1. On the one hand, traditional e-commerce platform actively embraces the interactive tool of live broadcast, and "e-commerce + live broadcast" promotes the transformation of graphic and text shelf e-commerce to live e-commerce; On the other hand, the entertainment social platform tries to realize the live broadcast traffic with the power of e-commerce, and "live + ecommerce" expands the marketing functions beyond the broadcast entertainment and information attributes. Whether "e-commerce + live" or "live + e-commerce", live e-commerce is still at the core of e-commerce in the final analysis, while live broadcast is one of the new ways for businesses to explore new transformation and flow realization. Live video business still cannot be separated from the combination of three elements of "people, goods and field". Different from that, live video operators innovate people and the field, integrate them into KOL host, MCN institutions and other participants, enrich marketing scenarios on the basis of "commodity details page", and promote closer integration of three elements. Compared with traditional e-commerce, the optimization of live e-commerce on "people, goods and field" drives the upgrade of user shopping experience, and has obvious advantages in marketing effect and user transformation [1]. 
Table 1. Comparative analysis of live e-commerce and traditional e-commerce

\begin{tabular}{|c|c|c|}
\hline Difference & $\begin{array}{l}\text { Live E- } \\
\text { commerce }\end{array}$ & $\begin{array}{l}\text { Traditional e- } \\
\text { commerce }\end{array}$ \\
\hline $\begin{array}{c}\text { Content } \\
\text { presentation }\end{array}$ & $\begin{array}{c}\text { Interaction } \\
\text { between KOL } \\
\text { and fans }\end{array}$ & $\begin{array}{c}\text { Graphic } \\
\text { information }\end{array}$ \\
\hline $\begin{array}{c}\text { Attribute } \\
\text { characteristics }\end{array}$ & $\begin{array}{l}\text { Entertainment + } \\
\text { online marketing }\end{array}$ & Offline marketing \\
\hline Business logic & $\begin{array}{l}\text { Anchor presents } \\
\text { product }\end{array}$ & $\begin{array}{l}\text { Users search } \\
\text { products by } \\
\text { themselves }\end{array}$ \\
\hline $\begin{array}{l}\text { Commodity } \\
\text { price }\end{array}$ & Have advantages & No advantage \\
\hline $\begin{array}{c}\text { Interactive } \\
\text { quality }\end{array}$ & Strong & Weak \\
\hline Conversion rate & Higher & Lower \\
\hline
\end{tabular}

Source: 36 krypton

In terms of theoretical significance, (1) in terms of research content, through literature review, the author finds that few studies focus on the detailed analysis of this topic from both sides of supply and demand. This paper analyzes the live broadcast e-commerce industry from a more comprehensive perspective and presents it with data charts. (2) In terms of research methods, this paper uses literature review, chart analysis, data analysis, case analysis, country comparison and other academic methods for analysis.

In terms of practical significance, under the epidemic situation, "home economy" has given birth to new players and new business opportunities in ecommerce live broadcasting industry. In 2019, due to the rapid rise of e-commerce live broadcasting, the live broadcasting industry will once again become the focus of attention and the outlet of the Internet industry; In 2020, due to the spread of the epidemic, the problems of delayed return to work and production and so on will further promote the development of e-commerce live broadcasting ecology. Compared with the past, the scope, field and importance of live broadcasting ecommerce participation have undergone tremendous changes. Not only all platforms and enterprises have increased investment in live broadcasting, but also the structure of anchor and the scale of users are growing rapidly.

\section{DEVELOMENT OF LIVE BROADCAST E-COMMERCE INDUSTRY}

\subsection{Development process and characteristics}

Since the development of China's Internet live broadcasting in 2005, it has gone through different processes from media transformation to content transformation and then to commercial realization transformation, and has entered the stage of 5.0 [2].

Live 1.0: the rise of Webcast. The time was from 2005 to 2011. It evolved from 9158 video website to create live show mode. Then, six rooms, YY and other platforms launched live show business. During the live show 1.0 period, it mainly used PC as the media and live show as the content.

Live 2.0: the content is turned on vertically. The time node is 2012. At this time, the domestic online game market is developing rapidly, and the live broadcasting platform with game content, Huya, is online, which marks the beginning of the era of game live broadcasting; In 2014, live Betta was launched, which promoted the further expansion from live show to live game in vertical field. The live media is still mainly PC.

Live 3.0: the rise of mobile live broadcasting. In 2015, 4G commercial landing promoted the explosive development of domestic mobile Internet, and network applications such as games, video and live broadcast were transferred to the mobile terminal, starting the live broadcast 3.0 stage. Compared with PC, mobile live broadcasting has the advantages of multi device, multi scene, fragmented time and the optimization of mobile network environment; With the help of capital and policy support, a large number of content creators UGC have entered the field of live broadcasting [3]. The content of entertainment live broadcasting shows a rich and diversified trend, and the wave of nationwide live broadcasting begins. At this stage, webcast has gradually completed the media transfer from PC to mobile and the transformation from show live to live + pan entertainment. The number of users has increased rapidly to 340 million in 2016, which is also known as the first year of mobile live.

Live 4.0: refined operation and live + enabling. With the rapid development of the industry, the number of mobile webcast platform apps has increased rapidly, once reaching $200+$, and the industry competition has intensified; At the same time, due to the extensive horse racing enclosure in the early stage of the industry, the non-standard content of some pan entertainment live broadcasting platforms and the existence of vicious competition, the regulatory authorities then issued a series of policies to strengthen the supervision of the 
content, anchor, platform and other links and to rectify the industry. Internal and external factors jointly drive the industry to integrate and clear up, small and medium-sized live broadcasting companies shuffle out, and the traffic, network, network, and other aspects of the industry Anchor and capital gathered to the head platform, and the industry competition pattern gradually moved to the giant competition [4]. In the competition of mobile Internet traffic, content is the king, which drives each platform to the direction of refined operation; With the new media attribute of strong interactivity, strong real-time and rich forms of expression, the content of live broadcast penetrates into various vertical fields such as e-commerce, sports, information, education, finance and business services, and derives more abundant cash flow modes, including live broadcast reward, advertising marketing, member income, game promotion, Commission sharing, etc.

Live 5.0: Live e-commerce broke out. In 2019, with the rise of live broadcasting with goods, the user scale shows explosive growth, and the high traffic conversion rate promotes the development and growth of ecommerce live broadcasting market, becoming an important part of the live broadcasting field after pan entertainment live broadcasting. Under the catalysis of the epidemic in 2020, the penetration of live broadcasting into different offline operation based industries will be accelerated, the ecological territory of e-commerce live broadcasting will continue to expand, and the national live broadcasting will move to the stage of national live broadcasting with goods. Different from the pan entertainment live broadcast reward as the main cash flow mode, the core income of live broadcast ecommerce cash flow mainly comes from the Commission sharing after the sale of goods, which is jointly carried out by the anchor, MCN institutions, platforms, etc.

\subsection{Driving force of development}

\subsubsection{Platform support and preferential policies}

For businesses, the promotion of the platform is one of the reasons to attract them to open live broadcast. More importantly, live broadcast can improve the user stickiness and potential revenue increment of businesses. Through live real-time interaction, businesses can reach consumers efficiently, greatly shorten the decision-making time of consumers, and stimulate the generation of consumer demand. In addition, the preferential commodity price in the live broadcast scenario has a certain attraction to consumers [5]. Under the stimulation of price drop, coupons, lucky draw and other preferential policies, consumer surplus is formed. In addition to the commodity labels such as "limited quantity", "starting in the live broadcast room" and the anchor's own "grass planting" ability, consumers'; surplus psychology can easily be transformed into purchasing behavior. As shown in Figure 1, there are more core users in the third tier and below cities in Taobao live broadcast, which are highly price sensitive and highly dependent on live broadcast products.

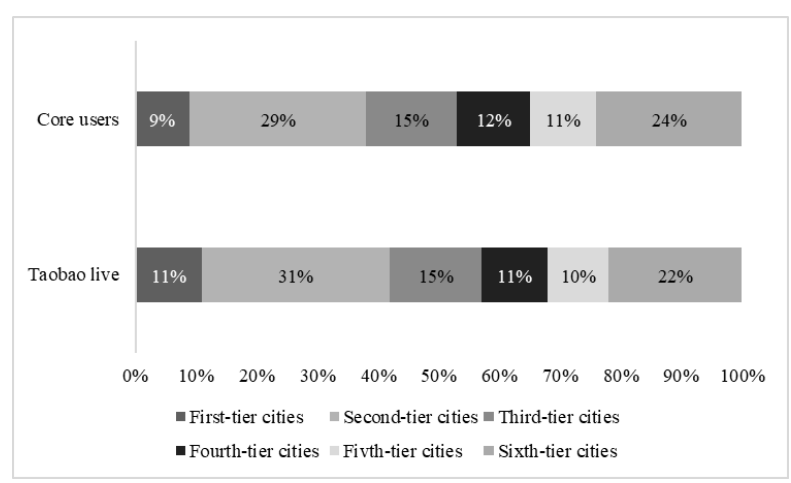

Figure 1: structure distribution of China's Taobao live broadcast and core users in first tier to sixth tier cities

Data source: Taobao live

In 2020, the novel coronavirus pneumonia outbreak came suddenly, and businesses under the line were frustrated and stock was overloaded. Live broadcast, a low-cost customer channel, is favored by businesses. Businesses are switching to live broadcasting online

Inventory, ease the pressure of cash flow. According to CNNIC statistics, in the first half of 2020, the total number of live broadcasts by businesses exceeded 10 million, with an average of nearly 1.5 million live broadcasts per day and 50 billion viewers.

During the epidemic period, each platform also increased support for live broadcasting, reduced technical service fees for businesses, optimized the settlement process of businesses, and encouraged offline businesses to open live broadcasting marketing mode; Open the traffic support plan to help businesses gain exclusive traffic; Training for the anchor to improve the level of the anchor with goods; Subsidizing some commodities, reducing or exempting Commission, etc [6]. The platform opens the door of live marketing for offline businesses in deep trouble from the aspects of fees, subsidies, settlement process, anchor training, etc.

\subsubsection{High quality talents}

With the rapid development of economy, a number of KOL talents with network influence have been born in China. KOL through its own influence and unique advantages, has formed a certain scale of fan groups on the purchase and transaction of accurate and efficient marketing. KOL, with its own characteristics of high conversion rate, has gradually developed into the core element "people" in live e-commerce, that is, the anchor. The formation of Internet celebrity culture and 
the penetration of KOL influence also drive the vigorous development of live e-commerce.

Internet Celebrities attract consumers to purchase by virtue of good fan relationship, high-quality oral marketing level or excellent personality charm. Its grass marketing advantages not only attract the attention of the platform, but also attract the attention of government departments. Especially during the epidemic period, the advantages of carrying goods and transforming consumption on the online red line are highlighted. The government's talent incentive to KOL promotes the market to cultivate more excellent live broadcast talents, and at the same time, it also injects a "heart tonic" into the development of live broadcast e-commerce.

\subsection{Market size analysis}

China's online shopping has been showing rapid growth, and has become an important driver of domestic consumption growth. At the same time, live broadcast ecommerce GMV shows a high-speed growth trend, which has increased from 19 billion CNY in 2017 to 433.8 billion $\mathrm{CNY}$ in 2019. Taking Taobao live broadcast platform as an example, Taobao live broadcast has guided the transaction growth of more than $150 \%$ for three consecutive years, and GMV will break out in full in 2019, reaching more than 200 billion CNY. Under the influence of the epidemic, the multi industry "cloud back to work" and consumers'; enthusiasm for "cloud shopping and cloud shopping" have boosted the evolution of this mode. It will continue to maintain high-speed growth and accelerate the penetration into the whole field of online shopping, which is expected to reach 961 billion CNY by 2020 .

With the rapid development of live broadcasting industry, the penetration rate of Internet users has reached $62 \%$ in March 2020, and the number of live broadcasting users has reached 560 million, including 265 million e-commerce live broadcasting users, accounting for $47.32 \%$ of the number of live broadcasting users. On the other hand, the penetration of online shopping in consumers'; daily life is more indepth [7]. By March 2020, the number of mobile online shopping users has reached 707 million, with a penetration rate of $78.9 \%$. Compared with this, the proportion of e-commerce live users is only $37.48 \%$, and the incremental space brought by user transformation is very broad. As shown in Figure 2, as of December 2020, the number of China's webcast users has reached 617 million, with a utilization rate of $62.4 \%$.

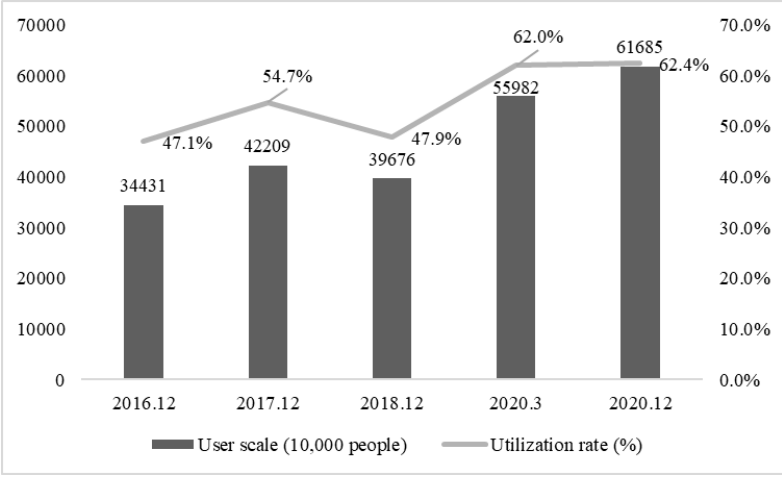

Figure 2: User scale and utilization rate of China's webcast from December 2016 to December 2020 $(10,000$ people; $\%)$

Data source: CNNIC Internet survey report

\section{ECOLOGICAL ANALYSIS OF LIVE E- COMMERCE INDUSTRY}

\subsection{Industrial chain structure}

In the ecology of live e-commerce, the upstream is mainly brands, dealers or manufacturers, the midstream is mainly MCN institutions, anchors and platform channels, and the downstream is consumers. According to the characteristics of the products, the brand companies launch commercial products to MCN organizations or anchors. MCN organizations provide incubation, promotion and management services for anchors. KOL outputs content and reaches consumers through the platform to complete the delivery. In addition, data marketing service providers provide data marketing services for brands and $\mathrm{MCN}$ institutions, such as screening KOL, formulating and implementing KOL live delivery scheme, supply chain service providers provide stable source of goods and product selection services for anchors, and integrated technology solution providers provide live broadcast technology and e-commerce technology services for live broadcast e-commerce platform.

\subsection{Industry participants}

\subsubsection{Live broadcast platform}

In the live broadcast e-commerce industry chain, the platform is responsible for building and maintaining scene services, and formulating relevant rules for participants to comply with. Therefore, all parties who want to bring goods on the platform need to comply with the corresponding rules of the platform, and the platform has the dominant power in the industry chain.

In the live e-commerce ecosystem, platforms are mainly divided into three categories: traditional ecommerce platforms represented by Taobao; The 
entertainment content platform represented by Tiktok. Shopping guide community platform represented by Xiaohongshu. The logics and emphases of the three types of platforms are different.

The driving force of the transformation from traditional e-commerce platform to live e-commerce is to expand the customer acquisition channels by live broadcasting, harvest the traffic and maintain certain user stickiness at the same time; The entertainment content platform has a rich flow pool, and it is an attempt and exploration for the platform to realize the flow through live broadcast into the e-commerce industry; In the shopping guide community platform, the mission of the shopping guide is to plant grass and reach the content, so it has the advantage of natural interaction with consumers [8]. It is natural to consolidate the e-commerce business through strong interactive live broadcast.

The differences among the three platforms determine their advantages and disadvantages. Traditional platform commodities and shopping guide community platform SKU are rich, the supply chain is relatively stable, users have strong purchasing mind, but the flow is not as good as entertainment content platform; Although the latter is dominated by traffic, the fans' minds are mostly entertainment oriented and the conversion rate is low.

Table 2: comparative analysis of three types of live broadcasting platforms

\begin{tabular}{|c|c|c|c|}
\hline Features & $\begin{array}{c}\text { Traditional } \\
\text { e-commerce } \\
\text { platform }\end{array}$ & $\begin{array}{c}\text { Entertainm } \\
\text { ent content } \\
\text { platform }\end{array}$ & $\begin{array}{c}\text { Shopping } \\
\text { guide } \\
\text { community } \\
\text { platform }\end{array}$ \\
\hline $\begin{array}{c}\text { Anchor } \\
\text { type }\end{array}$ & $\begin{array}{c}\text { Business self } \\
\text { broadcast }\end{array}$ & $\begin{array}{c}\text { Talent } \\
\text { anchor }\end{array}$ & $\begin{array}{c}\text { Anchor of } \\
\text { shopping } \\
\text { guide }\end{array}$ \\
\hline $\begin{array}{c}\text { User } \\
\text { portrait }\end{array}$ & $\begin{array}{c}\text { Consumptio } \\
\mathrm{n}\end{array}$ & $\begin{array}{c}\text { Entertainme } \\
\text { nt }\end{array}$ & $\begin{array}{c}\text { Growing } \\
\text { grass and } \\
\text { shopping }\end{array}$ \\
\hline $\begin{array}{c}\text { Transact } \\
\text { ion path }\end{array}$ & $\begin{array}{c}\text { Platform self } \\
\text { transaction }\end{array}$ & $\begin{array}{c}\text { Jump to } \\
\text { third party } \\
\text { E-commerce }\end{array}$ & $\begin{array}{c}\text { Platform self } \\
\text { transaction }\end{array}$ \\
\hline $\begin{array}{c}\text { Conversi } \\
\text { on rate }\end{array}$ & $\begin{array}{c}\text { Medium to } \\
\text { high }\end{array}$ & Lower & Higher \\
\hline $\begin{array}{c}\text { Typical } \\
\text { platform }\end{array}$ & Taobao & Tiktok & $\begin{array}{c}\text { Quotations } \\
\text { from } \\
\text { Chairman } \\
\text { Mao Zedong }\end{array}$ \\
\hline
\end{tabular}

\subsubsection{MCN mechanism}

MCN, or multi-channel network, originated from the linking platform between Youtube and professional content creators. After the related concepts entered China, the functions of MCN have been continuously expanded, integrating the abilities of content creation, traffic introduction and exposure, commercial cash flow, etc. on the basis of the original creator aggregation function.

Since 2015, the number of MCN institutions has been growing rapidly, from 150 in 2015 to more than 20000 in 2020.The background of new players is diverse: the transformation of traditional film and television entertainment companies; $\mathrm{MCN}$ organization built by stars; $\mathrm{MCN}$ organization is set up in the enterprise; Internet investment in MCN institutions [9].

After four years of rapid development, China's MCN has gradually formed three types of organization: Pan entertainment type, information type and live broadcast e-commerce type, covering seven types of business, including content production, operation, marketing, brokerage, knowledge payment, copyright and live broadcast. The main cash flow mode of entertainment MCN institutions is advertising marketing; Information based MCN organizations use knowledge payment as their revenue source; The live broadcast e-commerce MCN institutions share Commission with the platform for commercial realization.

Since 2019, the outbreak of live broadcasting ecommerce has driven MCN institutions to increase their live broadcasting format. According to topklout's survey of China's major MCN institutions in 2020, about $58 \%$ of the institutions indicated their determination to make efforts to live broadcast ecommerce in the future.

In the live e-commerce ecosystem, $\mathrm{MCN}$ institutions play a more "intermediary" role in cultivating anchors throughout their life cycle and delivering high-quality anchors to the platform.

\subsection{Business model and value}

In the live e-commerce ecosystem, businesses, platforms and MCN institutions / anchors participate in sharing based on transaction volume. The determination of commission amount is mainly based on CPS mode, that is, cost per sales, with transaction rated Commission. Generally, the Commission is $20 \%$ of GNV. The Commission is shared by live broadcast platform, third-party cooperation platform and MCN organization / anchor, and each live broadcast platform has different division of commission distribution proportion. Take Taobao as an example. In the noncooperative state, $30 \%$ of the Commission, i.e. $6 \%$ of GMV, flows into Ali's marketing platform, Ali mom and Ali creative platform, and the remaining $70 \%$ of the Commission is distributed by MCN organization and anchor; Tiktok and Kwai, such as the three party 
platform cooperation scenario, Taobao charged $7.4 \%$ of GMV as content scenario and technical service fee, the remaining Commission belonged to the cooperation platform and MCN agency / anchor. Suppose (1) the merchant sets aside $20 \%$ of the total transaction amount as the Commission, and (2) the MCN shares 5:5 with the network red anchor. According to the current rules, after the author's calculation, the final proportion of the Commission distribution is as follows:

Table 3: Commission sharing proportion of each participant in live broadcasting and delivery

\begin{tabular}{|c|c|c|c|}
\hline $\begin{array}{c}\text { Participan } \\
\text { ts }\end{array}$ & $\begin{array}{c}\text { Taobao } \\
\text { live }\end{array}$ & Participants & Tiktok \\
\hline $\begin{array}{c}\text { Ali } \\
\text { Department }\end{array}$ & $6 \%$ & $\begin{array}{c}\text { Ali } \\
\text { Department }\end{array}$ & $7.4 \%$ \\
\cline { 3 - 4 } & & $\begin{array}{c}\text { Live } \\
\text { broadcast } \\
\text { platform }\end{array}$ & $6.3 \%$ \\
\hline MCN & $7 \%$ & MCN & $3.15 \%$ \\
\hline anchor & $7 \%$ & anchor & $3.15 \%$ \\
\hline
\end{tabular}

\section{TYPICAL CASE ANALYSIS OF LIVE E- COMMERCE INDUSTRY: TAOBAO}

Perfect e-commerce ecology. The vast majority of Amoy products reach the consumers by entering the live broadcast room. By the end of 2019, the commodity types of Taobao live broadcasting room increased by $190 \%$ over the same period last year, covering all kinds of commodities such as home appliances, automobiles, food, 3C digital, etc. At the same time, Taobao has cooperated with more than $1600 \mathrm{MCN}$ organizations and more than 200 live broadcast agencies to establish 100 clothing bases and 17 jewelry bases, greatly enriching the connotation of "people, goods and market".

Consumer oriented user mind and efficient platform governance level. Since the live broadcast entrance was opened, Taobao has established a series of live broadcast specifications to restrict live broadcast behavior and protect the rights and interests of consumers and businesses. Recently, Taobao has also opened up social supervision channels, and used scientific and technological means such as AI to detect the live content, so as to create a healthy live ecology.

\section{ANALYSIS ON THE DEVELOPMENT TREND OF ONLINE RED LIVE BROADCASTING AND E-COMMERCE LIVE BROADCASTING}

\subsection{Development Suggestions}

All parties work hand in hand to integrate highquality resources by means of refined operation to create a "tree" health industry ecology. All kinds of bonus blessings, such as traffic, consumption habits and policies, accelerate the penetration of live e-commerce into various consumption circles. However, behind the rapid development of the industry, pain points are emerging: the good and bad of live broadcast products are intermingled, damaging the rights and interests of consumers; Human operation of live data infringes the interests of businesses. In view of the current situation of the undercurrent industry, platforms, MCN institutions, businesses and regulators need to work together to gather high-quality resources, realize refined operation, and create a healthy and stable live ecommerce ecology [10].

In order to construct sustainable development ecology, the following elements are the core and key: private flow, flexible supply chain, high-quality talents and industry constraints. Four elements converge to the live broadcast e-commerce industry to promote the formation of "tree" healthy ecology of the industry: supervision is the root, which is the basis of ecological sustainability, and is supported by the strong risk monitoring and control ability of regulatory agencies; Flexible supply chain and private flow pool are the branches and the key to industry competition, which can not be separated from the refined management of "people, goods and market" by businesses and MCN institutions; Talents play the role of branches and leaves, adding icing on the cake to live e-commerce.

\subsection{Industry Trend Outlook}

5.2.1 The three elements of "people, goods and market" extend to the direction of diversification to expand the value boundary of live broadcast e-commerce

Commodity diversification. The types of live broadcast commodities are from light decision-making commodities to high customer price and heavy decisionmaking commodities, and the types of live broadcast SKUs are more abundant and perfect. Real estate finance, $3 \mathrm{C}$ digital, online education and other products will further enter the studio"The era of "everything can be broadcast live" is just around the corner.

There are various types of anchors. The joining of celebrities in politics, business, entertainment and other 
fields has promoted the diversification of anchor types. The entry of virtual idol into the live broadcasting room will break the circle of users and accelerate the penetration of live broadcasting e-commerce.

The live content is diversified. The boundary between variety show and live broadcast will be more and more blurred. E-commerce platforms and streaming media platforms have created "variety like" live marketing programs to attract the younger generation of users and enhance their stickiness. At the same time, they also potentially promote the transformation from online grass planting to offline consumption.

Live scenes are diversified. Live broadcast to help farmers and special live broadcast to the Expo are now available. In the future, there will be more scenarios integrated with live e-commerce, allowing users to "buy while learning", "buy while playing" and "buy while doing public welfare".

Various forms. The combination of instant live broadcast and retained short video or graphics and text can make the live broadcast win customers, and the short video / graphics and text can realize the further retention and precipitation of users.

\subsection{2 $5 G$ enabling is promising, immersive, interactive and high-definition live shopping experience is just around the corner}

"New infrastructure" has accelerated the implementation and popularization of $5 \mathrm{~g}$. Since China officially launched $5 \mathrm{~g}$ commercial service in November 2019, a total of $6900005 \mathrm{~g}$ base stations have been built by the end of September 2020, basically realizing the coverage of prefecture level.5g has three characteristics of high speed, low delay and wide connection, which become the underlying technology foundation of $4 \mathrm{~K} /$ $8 \mathrm{~K}$ ultra high definition video and VR / AR applications. The optimization and integration of various technologies will inject new energy into live broadcast e-commerce.

Say goodbye to blur and embrace Ultra HD vision. Affected by the transmission efficiency of live broadcast equipment and $4 \mathrm{G}$ network, live broadcast pages are often blurred, which affects the intuitive and comprehensive display of product details.5g makes it possible for $4 \mathrm{~K} / 8 \mathrm{~K}$ ultra high definition live broadcasting to meet consumers' expectations for high quality and fluency of live broadcasting and optimize consumers' shopping experience.

Broadcast everywhere, live scenes tend to be diversified.5g high transmission rate and high mobility, is expected to continue to enrich the live scene. In particular, with the improvement of coverage, $5 \mathrm{~g}$ provides a huge imagination for live broadcast scenes of origin and factories in remote areas, shortens the distance between commodity production and consumers, and imperceptibly drives the transaction.

Immerse yourself and enhance the holographic immersive shopping experience. The integration of VR / $\mathrm{AR}$ and live e-commerce brings users "immersive" consumption experience. In the VR / AR live scene, users' online make-up and fitting has gradually changed from imagination to reality.

\section{CONCLUSION}

\subsection{Summary}

Live e-commerce reconstructs the three elements of "people, goods and market" by means of live broadcasting, but its essence is still e-commerce. Compared with traditional e-commerce, live ecommerce has the advantages of strong interaction and high conversion rate.

Since the online live shopping function of ecommerce platform in 2016, live e-commerce has developed rapidly, and the outlet has appeared. From 2016 to 2019, the average annual growth rate of live broadcast e-commerce market size will be maintained at more than $200 \%$. The vigorous development of the industry is inseparable from the cultivation of consumers' live viewing habits, the vigorous promotion of various e-commerce platforms, the exploration of business revenue channel expansion, the formation of KOL red culture and various policy support.

After five years of rapid development, the ecology of live e-commerce has been gradually improved: the midstream platform, MCN institutions and anchors have realized the link between upstream businesses and downstream consumers. MCN institutions mainly play the role of "intermediary" to transport anchor resources for the platform. With the continuous improvement of the penetration rate of live e-commerce, the trend of cross-border anchor becomes more and more obvious. Celebrities and virtual idols have entered live ecommerce.

Behind the rapid development of the industry, pain points are gradually emerging. To build the health industry ecology, all parties need to work together to achieve fine operation and gather high-quality resources.

With the bonus of funds and policies, the live broadcast e-commerce is expected to achieve further growth, and the industry will gradually develop in the direction of standardization and orderliness. In the future, with the growing number of participants, the three elements of "people, goods and market" will become more and more diversified, and live broadcast products and content, anchor types, live broadcast scenes and marketing forms will be enriched to expand 
the value boundary of live broadcast e-commerce. With the optimization of $5 \mathrm{~g}$ technology, a more interactive, real and high-definition online shopping experience can be realized, bringing more imagination to the live ecommerce industry.

\subsection{Expectation}

Due to space constraints, this paper fails to give a detailed description of the private traffic of live broadcasting and the development of live broadcasting e-commerce in low tier cities. In the future, the author will make a further in-depth analysis along this direction.

\section{REFERENCES}

[1] CHENG Ye, WANG Rui. Governance Mechanisms of Live Streaming Platform :Case Studies from the Two-sided Platform Perspective[J]. journal of management case studies, 2017.

[2] Wen-Sheng Z, Jie W U, Hui-Min X. An Analysis of Current Development of E-commerce Live Broadcast and Relevant Strategies[J]. Journal of Jilin Teachers Institute of Engineering and Technology, 2017.

[3] Gerard C Johnson, Sean Bunner, John McDevitt. Method and system for improved E-commerce shopping: US 2013.
[4] Huijuan X. Analysis on the Present Situation and Problems of Taobao Live Broadcasting[J]. Office Informatization, 2019.

[5] Yu-Qi L. Research on the Web Celebrity Economy Marketing Model from the Background of We Media[J]. Value Engineering, 2018.

[6] Wen-Ye Z, Xi-Xi L I, Shu-Hong G, et al. Analysis of the Development Mode of Internet Celebrity Economy[J]. Management \& Technology of SME, 2018.

[7] Ying-Xian P, Yu-Wen Z, Bing-Bing L, et al. SWOT Analysis of Self Media Development from the Perspective of Internet Celebrity Economy[J]. management \& technology of sme, 2017.

[8] Haihua W, Yu L I, Lijun X, et al. Influence of Live Web Celebrity Attributes on Users Value Cocreation[J]. Shanghai Management ence, 2019.

[9] DANIEL, KAHNEMAN. Maps of bounded rationality: psychology for behavioral economics[J]. American Economic Review, 2003.

[10] Day R H. Behavioral economics: implications for economic theory and policy[J]. Journal of socioeconomics, 2004, 33(6):p.715-724. 